\title{
Public awareness of oral cancer and associated risk factors is low
}

\author{
Abstracted from \\ Grant E, Silver K, Bauld L, Day R, Warnakulasuriya S. \\ The experiences of young oral cancer patients in Scotland: \\ symptom recognition and delays in seeking professional help. Br Dent J 2010; 208: 465-471. \\ Address for correspondence: Saman Warnakulasuriya, \\ Department of Oral Medicine and Pathology, King's College London Dental Institute, \\ Bessemer Road, London, SE5 9RS. E-mail: s.warne@kcl.ac.uk
}

\section{Question: What are the reasons for delay in seeking help from a clinician among young oral cancer patients?}

Study design A survey was carried out over a three-year period (2004-2007) in Maggie's Cancer Caring Centres or in patients' homes in Glasgow and Edinburgh, Scotland. Participants included young patients diagnosed with oral cancer.

Data collection and analysis Data were collected by interview using a semi-structured interview schedule. The interview transcripts were analysed using a thematic framework and with the aid of NVivo qualitative analysis software (Version 8).

Results The majority of the cohort knew that smoking and alcohol could cause oral cancer. None thought it would happen to them, however. Descriptions of symptoms varied widely and several participants used self-treatment modalities provided from a pharmacy. There were various causes of patient delay, and self-treatment was not the only cause. Reinterpretation of symptoms without seeking professional help was not uncommon. None of the patients suspected that they had oral cancer until it was confirmed by their general practitioner (GP) or general dental practitioner (GDP).

Conclusions The study confirms gaps in understanding and awareness of oral cancer. Most survey participants had heard of oral cancer. However, they did not think their symptoms were indicative of cancer and they self-managed the problem. The culture of not bothering the GP/GDP unless the condition was perceived as serious is a barrier to early diagnosis and treatment. Findings support that further public awareness of oral cancer and its symptoms is required to alert the public that if their symptoms persist beyond three weeks, they need a professional opinion.

\section{Commentary}

The incidence of oral cancer continues to rise in the UK, especially in Scotland. Most of these cancers are diagnosed at advanced stages, resulting in extensive treatments, impaired function and poor outcomes. Early diagnosis remains an important predictor of prognosis and survival.

The aim of this study was to examine the responses of young oral cancer patients in Scotland to the symptoms of their emerging condition, to understand the ways they seek help and to investigate delay caused by not recognising symptoms associated with oral cancer. This work is the first detailed investigation focusing exclusively on the experiences of young oral cancer patients, their symptom recognition and paths to referral, in Scotland.

The study included 15 patients under 45 years of age, who were diagnosed with oral cancer in the three years before the study and resided in central Scotland. The most common cancer site was the tongue, as it was reported in seven participants. In addition, the study population included comparable numbers of men and women, seven and eight, respectively.

Interviews were conducted using a semi-structured interview schedule, which was initially piloted on adult volunteers among oral cancer patients known to the study organisers. Furthermore, a timeline was drawn up for each interviewee to maintain a picture of the chronology of significant events and to enable some analyses of reported delays.

To better understand the findings of this study as well as factors pertaining to delays in seeking care, the results will be discussed in light of the Health Belief Model. This model was first developed in the 1950s by a group of U.S. Public Health Service researchers who wanted to explain why so few people were participating in programs to prevent and detect disease. ${ }^{1}$ Subsequent amendments to the model were made as late as 1988. The model addresses the individual's perceptions of the threat posed by a health problem (susceptibility, severity), the benefits of avoiding the threat and factors influencing the decision to act (barriers, cues to action and self-efficacy). ${ }^{2}$ The following six constructs of the Health Belief Model will be used to examine the perceptions and actions of study participants in terms of controlling their illness.

- Perceived susceptibility is the individual's assessment of their risk of getting the condition. In this study, several interviewees, even those who were aware of oral cancer, said that they had not expected their own diagnosis and did not think their symptoms were indicative of cancer.

- Perceived severity refers to the patient's belief that the condition has serious consequences. Most study participants $(n=12)$ indicated that they did not, at first, think their symptoms were serious. Most assumed they had a minor condition, such as an abscess or ulcer.

- Perceived benefits of taking action to reduce susceptibility to the condition or its severity were not explicitly discussed in this study. Nonetheless, patients' perception that seeing a clinician may not improve their condition or save care costs over time is a common phenomenon in clinical practice. In the case of oral cancer patients, it is reasonable to assume that such perception may lead to delayed diagnosis and treatment. 
- Perceived barriers The survey demonstrated the presence of a culture of not wanting to bother the GP/GDP unless a condition was really serious. The concern about 'wasting the time' of a health professional was a factor in delaying earlier access to care for some of the participants. Additional barriers may have included systemrelated factors, such as access to care and waiting lists.

- Cues to action refer to exposure to factors that prompt seeking care. Among study participants who had some prior knowledge of the disease, several had remembered seeing a TV campaign, which was developed as part of the West of Scotland Cancer Awareness Programme. For some interviewees, seeing this TV feature had led them to make an initial appointment with a health professional to examine their symptoms.

- Self-efficacy is demonstrated when patients are confident in their ability to successfully perform an action to alleviate their condition. Most interviewees reported that they attempted some form of selftreatment before seeking help by purchasing over the counter remedies. This action may be considered a form of self-efficacy. At the same time, it can be argued that most participants lacked self-efficacy since they did not make an early decision to visit a healthcare professional despite their prior knowledge of oral cancer.

The study indicated that most participants had some awareness of oral cancer before noticing their own initial symptoms, and this prior knowledge came from a range of sources. However, a few had not been aware of oral cancer at all before it happened to them. Most patients described awareness of two of the main risk factors for oral cancer, smoking and alcohol consumption, but their views about whether these behaviours were directly linked to their condition were mixed.

Due to the small sample size and the omission of some details by participants, gender was not used as a unit of analysis, but it was used to inform the analysis and interpretation of interview data. As for socio-economic status (SES), the only indicator that was collected for almost all participants was post code. An analysis of post code data using the Scottish Index of Multiple Deprivation showed that the sample was mixed, with several participants living in affluent areas. However, a growing body of evidence suggests that low SES plays a role in both the likelihood of developing the disease and the manner in which disadvantaged patients seek care. ${ }^{3}$
Findings from this study support previous research suggesting that public awareness of oral cancer and associated risk factors is low in the UK. Awareness of risks and symptom recognition among oral cancer patients are crucial factors in determining survival rates. Early detection was shown to improve the chances of survival and to reduce the likelihood of advanced staging of disease at diagnosis. ${ }^{4}$

Moreover, the work indicated that the understanding of the link between tobacco and alcohol use and oral cancer in Scotland remains weak. This is an important issue both for future research and health promotion campaigns. Initiatives such as the West of Scotland Cancer Awareness Programme are now more important than ever. The same can be said about social marketing campaigns to prompt individuals with symptoms to consult health professionals and to educate the public on the risk factors for oral cancer. Historically, most campaigns focused on tobacco use and heavy alcohol consumption as risk factors. Until recently, little or no public attention was given to the strong link between infection with the human papilloma virus (HPV) and oral cancer. A recent article from The Observer newspaper indicated that the $\mathrm{BBC}$ is preparing a documentary to investigate the link between HPV infection via oral sex and the rising numbers of oral cancer cases among youths in Britain. ${ }^{5}$

Finally, although the findings of the study are not generalisable to young oral cancer patients at large, some of the similar experiences of study participants can be identified as important themes that guide future research. Furthermore, due to elevated incidence in the UK, the role of dentists and family physicians in the early detection of oral cancer is becoming increasingly important. As such, opportunistic screening of patients by care providers should be encouraged.

\section{Imad AI-Dakkak}

\section{Centre for Evidence-based Dentistry, Oxford, UK}

1. Hochbaum, G. Why people seek diagnostic X-rays. Public Health Rep 1956; 71: 377-380.

2. Rosenstock IM, Strecher V], Becker MH. Social learning theory and the health belief model. Health Educ Q 1988; 15: 175-183.

3. Conway DI, McMahon AD, Smith K,et al. Components of socioeconomic risk associated with head and neck cancer: a population-based case-control study in Scotland. Br J Oral Maxillofac Surg 2010; 48: 11-17.

4. Gomez I, Seoane J, Varela-Centelles P, Diz P, Takkouche B. Is diagnostic delay related to advanced-stage oral cancer? A meta-analysis. Eur J Oral Sci 2009; 117: 541-546.

5. Campbell D, Thorpe V. Cancer risk to be tackled by documentary. The Observer, 17 October, 2010; p11.

Evidence-Based Dentistry (2010) 11, 106-107. doi:10.1038/sj.ebd.6400753 